\title{
Symbiogenics: An Epigenetic Approach to Mitigating Impacts of Climate Change on Plants
}

\author{
Claire Woodward \\ U.S. Geological Survey, Western Fisheries Research Center, Seattle, WA 98115; and the Biology Department, \\ University of Washington, Seattle, WA 98195 \\ Lee Hansen \\ Department of Chemistry and Biochemistry, Brigham Young University, Provo, UT 84602 \\ Fleur Beckwith \\ U.S. Geological Survey, Western Fisheries Research Center, Seattle, WA 98115; and the Biology Department, \\ University of Washington, Seattle, WA 98195 \\ Regina S. Redman \\ U.S. Geological Survey, Western Fisheries Research Center, Seattle, WA 98115; and the Biology Department \\ and Department of Forestry, University of Washington, Seattle, WA 98195 \\ Rusty J. Rodriguez ${ }^{1,2}$ \\ U.S. Geological Survey, Western Fisheries Research Center, 6505 NE 65th Street, Seattle, WA 98115; \\ and the Biology Department, University of Washington, Seattle, WA 98195
}

Additional index words. symbiosis, stress tolerance, fungal endophyte, plant microbe interaction, drought tolerance, salt tolerance

\begin{abstract}
We have found that plants in natural ecosystems adapt to abiotic stress by forming symbiotic associations with Class 2 fungal endophytes. Without the endophytes, plants are not stress-tolerant and do not survive in the habitats to which they are adapted. Symbiotically conferred stress tolerance typically occurs in a habitat-specific manner and is based on interactions between environmental factors and both plant and fungal genomes. For example, endophytes from geothermal plants confer heat tolerance, and endophytes from coastal plants confer salt tolerance. We have designated this phenomenon as habitat-adapted symbiosis and hypothesize that it is a ubiquitous aspect of plant ecology. Class 2 endophytes also increase plant growth and development while decreasing water consumption. We present metabolic and gene expression data to support a working model for the underlying mechanisms of endophyte-conferred benefits to plants. Although endophytes had no effect on photosynthetic rate and metabolic efficiency, they significantly increased photosynthetic efficiency. Endophytes significantly altered the ratio of upregulated to downregulated (UR:DR genes) plant genes compared with nonsymbiotic plants. Specific UR:DR gene ratios varied with endophyte species as well as habitat of origin. Collectively, these observations have allowed us to design a new symbiogenic (symbio = symbiosis; genic $=$ gene influence) strategy for mitigating impacts of climate change on crop production.
\end{abstract}

The greatest threats to agricultural sustainability in the 21 st century are drought, increasing temperatures, and soil salinization, all of which are being exacerbated by climate change (<http://www.copenhagendiagnosis. com $>$ ). Three approaches currently being

Received for publication 8 Dec. 2011. Accepted for publication 6 Mar. 2012.

This paper was part of the colloquium, "Emerging Techniques to Evaluate and Mitigate Crop Environmental Stress in a Changing Climate" held 28 Sept. 2011 at the ASHS Conference, Waikoloa, HI, and sponsored by the Environmental Stress Physiology (STRS) Working Group.

Funding was provided by NSF $(0414463,0950447)$, US/IS BARD (3260-01C), ARO (54120-LS), and USGS

We thank Jeff Duda, Yong Ok Kim, and Leesa Wright for assistance with statistical analysis, data collection, and helpful comments.

The use of trade, firm, corporation names, or products in this publication is for the information and convenience of the reader and does not constitute an official endorsement or approval by the U.S Government.

${ }^{1}$ Current address: Symbiogenics, Seattle, WA 98125

${ }^{2}$ To whom reprint requests should be addressed; e-mail rjrodriguez@symbiogenics.org. taken to develop stress-tolerant plants involve genetic modification, mutational selection, and breeding traits from wild plants (Tester and Bacic, 2005; Yamaguchi and Blumwald, 2005). However, these efforts have had limited field success presumably because 1) stress tolerance involves genetically complex processes; and 2) the ecological and evolutionary mechanisms responsible for stress tolerance in plants are poorly defined (Flowers and Flowers, 2005).

It is interesting that all plants are capable of sensing and responding to abiotic stresses such as salt, heat, and drought, but few plants are able to adapt and survive in habitats imposing these stresses (Perelman et al., 2007; Stout and Al-Niemi, 2002). The modern evolutionary synthesis states that stress adaptation is thought to occur by small genetic changes in an organism's genome that gradually occur over time (Mayr, 1993). This perspective is predicated on the assumption that complex organisms such as plants and animals exist as individuals. However, all plants and animals in nature are symbiotic with microorganisms and the number of microbial cells may be greater than the number of host cells.

The recent description of habitat-adapted symbiosis (Rodriguez et al., 2008) indicates that plants in high-stress habitats depend on Class 2 fungal endophytes (herein referred to as endophytes) for stress tolerance (Rodriguez et al., 2009b). Without the endophytes, the plants are unable to survive in the habitats to which they are adapted. Remarkably, the endophytes can infect, colonize, and communicate similarly with genetically divergent crop plants (monocots and eudicots) as they do with the native plants from which they were isolated. Therefore, endophytes from geothermal and coastal plants are able to confer heat and salt tolerance to crop plants such as cucurbits or tomato, respectively (Rodriguez et al., 2008). The ability of endophytes to confer stress tolerance to both monocots and eudicots suggests that the genetic basis of symbiotic communication is conserved and predates the divergence of these plant lineages $\approx 230$ MYA (Chaw et al., 2004; Wolfe et al., 1989; Yang et al., 1999). Because endophytes alter plant gene expression and phenotype without causing changes in the underlying plant DNA sequences and can be vertically transmitted between generations through seedcoats, these symbioses can be defined as epigenetic phenomena.

Collectively, endophytes can have profound effects on the physiology, growth, 
development, and reproduction of plants (Rodriguez et al., 2009b). Within $24 \mathrm{~h}$ of colonization, symbiotic (S) plants may show strong growth responses and during seedling development symbiotic plants preferentially develop extensive root systems before substantial shoot growth (Rodriguez et al., 2009a). Endophyte colonized monocot and eudicot seedlings consume up to $50 \%$ less water than nonsymbiotic (NS) controls (Redman et al., 2011; Rodriguez et al., 2008). A field study in southern Utah indicated that endophytes regulate sexual reproduction in sagebrush subspecies (Miglia et al., 2007; Rodriguez et al., 2009a). Endophyte-conferred disease resistance involves the activation of host defense systems more rapidly and to greater levels when symbiotic plants are exposed to virulent pathogens (Redman et al., 1999) compared with nonsymbiotic plants. Finally, high levels of stress induce metabolic imbalances in plants resulting in the generation of reactive oxygen species (ROS). Plants colonized with endophytes that confer tolerance to the applied stress do not produce ROS to levels that destroy membranes and chlorophyll (Apel and Hirt, 2004).

We present data to support a working model (Fig. 1) explaining the basis of endophyteconferred fitness benefits in crop plants and describe the potential of symbiogenic technology for sustaining agricultural productivity in the 21 st century.

\section{MATERIALS AND METHODS} tion. Oryza sativa L. ssp. japonica 'M-206' (rice) and Solanum lycopersicon 'Tiger-Like' (tomato) were chosen as study species to represent monocotyledon and dicotyledon crops of agricultural importance and each allows access to genomic sequence data. These varieties were also chosen because
Plant growth conditions and plant inocula-

they express growth responses and abiotic stress when colonized with endophytes, seeds are available commercially, and both grow well in the Pacific Northwest. Plants were grown under fluorescent lights of $70 \mu \mathrm{mol} \cdot \mathrm{m}^{-1} \cdot \mathrm{s}^{-1}$ set to a 12-h light cycle. Rice were surfacesterilized and germinated on $1 \%$ water agar media before being inoculated as described by Redman et al. (2011). Tomato seeds were surface-sterilized and grown on sterile vermiculite. Before the emergence of the first true leaves, plants were hydroponically inoculated with the following fungal endophytes at spore concentrations of $10^{4}$ spores $/ \mathrm{mL}$ : Colletotrichum magna L2.5 (L2.5), Colletotrichum magna R5 (R5), Curvularia protuberata 4666D (Cp4666D), Curvularia protuberata VF (CpVF), Fusarium culmorum Red1 (FcRed1), or Fusarium culmorum 18 (Fc18). After $3 \mathrm{~d}$ in inoculum or water (for the NS) controls, S and NS seedlings were transferred to double deckers (Rodriguez et al., 2008). C. magna wildtype isolate L2.5 forms an asymptomatic endophytic relationship in tomato (Redman et al., 2001) and R5 is a restriction enzyme mediated integration (REMI) mutant, which has a non-pathogenic lifestyle in tomato (Redman et al., 1999). C. protuberata isolate $\mathrm{Cp} 4666 \mathrm{D}$ was isolated from plants in geothermal soils and confers heat tolerance (Redman et al., 2002) and $\mathrm{CpVF}$ is an isogenic strain derived from Cp4666D that no longer confers heat tolerance (Márquez et al., 2007), and neither isolate confers salt tolerance (Rodriguez et al., 2008). $F$. culmorum isolate FcRed1 was isolated from plants growing on coastal beaches and specifically confers salt tolerance, isolate $\mathrm{Fc} 18$ was isolated from an inland plant and does not confer salt tolerance, and neither isolate confers heat tolerance (Rodriguez et al., 2008). Conditions for heat and salt stress were followed as described by Redman et al. (2011) and Rodriguez et al. (2008).

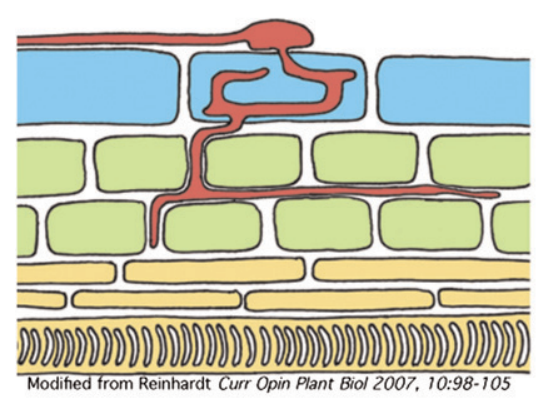

Endophytes grow in non-vascular apoplast

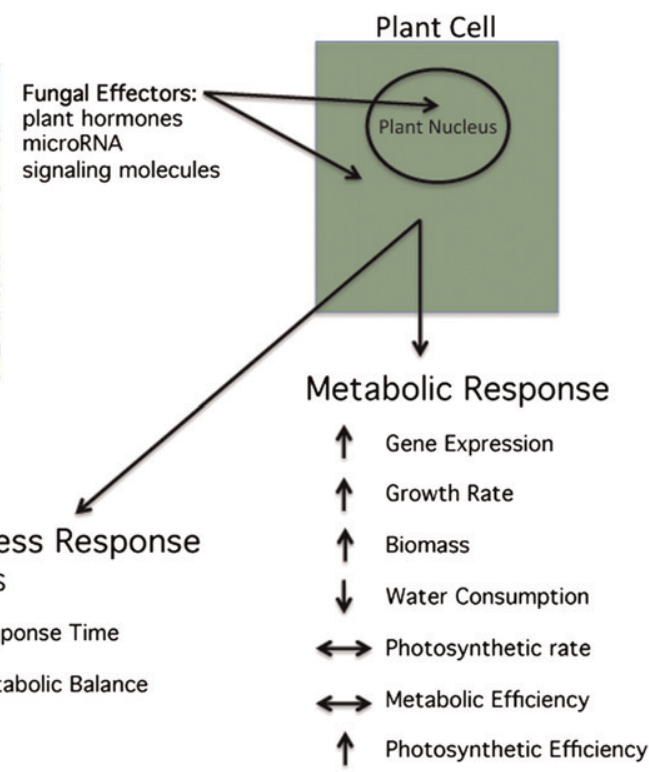

Fig. 1. Class 2 fungal endophytes as epigenetic regulators of plant metabolism. A working model of the communication between fungal endophytes and plant hosts.
Differential scanning calorimetry. Metabolic efficiency measurements were collected within $30 \mathrm{~min}$ of collecting leaf samples from $39 \mathrm{~S}$ and $39 \mathrm{NS}$ rice plants. Approximately $100 \mathrm{mg}$ of leaf tissue was removed with a razor blade and placed in an ampule of the isothermally operated calorimeter (Calorimetry Sciences Corporation MC-DSC Model 4100, Lindon, UT). The metabolic heat rate $\left(\mathrm{R}_{\mathrm{q}}\right)$ and rate of $\mathrm{CO}_{2}$ production $\left(\mathrm{R}_{\mathrm{CO} 2}\right)$ were measured and used to calculate metabolic efficiency by previously described methods (Criddle and Hansen, 1999; Criddle et al., 1997; Hansen et al., 2005). Dry weights were obtained on the samples used for metabolic measurements after drying in an oven at 70 to $80^{\circ} \mathrm{C}$ for $24 \mathrm{~h}$. Statistical analysis was done using a nonparametric $t$ test $(P>0.05)$.

Photosynthesis measurements. Photosynthetic rate, Fv/Fm, and ФPSII were measured using plants that had been light-adapted for at least $1 \mathrm{~h}$ by sampling the third leaf of 3week-old plants that were of equal size grown as described previously with a LI-6400 portable photosynthesis system (LI-COR, Inc., Lincoln, NE). Photosynthetic measurements were taken under the following conditions: temperature $25^{\circ} \mathrm{C}, \mathrm{CO}_{2}$ flow rate $400 \mu \mathrm{mol} \cdot \mathrm{s}^{-1}, \mathrm{CO}_{2}$ level $400 \mathrm{ppm}$, relative humidity $25 \%$ to $28 \%$, light intensity $500 \mu \mathrm{mol} \cdot \mathrm{m}^{-2} \cdot \mathrm{s}^{-1}(6400-02 \mathrm{~B}$ RedBlue \#SI-1537). Chlorophyll fluorescence measurements were made on NS and $\mathrm{S}$ tomato plants $(\mathrm{N}=5)$ aged 3 weeks and grown for $48 \mathrm{~h}$ with or without $250 \mathrm{~mm} \mathrm{NaCl}$. A time course experiment had been previously conducted monitoring stress response genes in both symbiotic and nonsymbiotic plants in response to stress. Forty-eight hours was chosen as a time point for further experiments because at this point, all the plants were responding to the stress but plant metabolism was being maintained to varying degrees. Statistical analysis was done with a $t$ test $(P<0.05)$.

Gene expression data. To determine if the fungal endophytes were acting as epigenetic regulators of plant genomes, gene expression patterns were measured with tomato microarrays, comparing NS with S tomato plants with and without stress. Symbiotic and NS tomato plants were grown without stress until they had produced four true leaves. At this point, half of the plants from each treatment were exposed to stress (salt or heat). The stress imposed on $\mathrm{S}$ and NS plants was determined by the habitatspecific stress tolerance of the particular endophytes used (Rodriguez et al., 2008). The remaining plants were maintained as unstressed controls. For salt stress experiments NS, FcRed1, and Fc18 were exposed to 200 mM salt for $24 \mathrm{~h}$ and plants were sampled at Times 0 and $24 \mathrm{~h}$ after application of $200 \mathrm{~mm}$ $\mathrm{NaCl}$. For heat stress experiments, NS plants and plants inoculated with $\mathrm{Cp}$ 4666D and CpVF were sampled at Times 0 and 48 hours after the plants were exposed to $45{ }^{\circ} \mathrm{C}$ heat (Rodriguez et al., 2008). Unstressed control plants and plants exposed to stress were sampled and immediately placed in liquid nitrogen and stored at $-80{ }^{\circ} \mathrm{C}$ until RNA 
extraction. RNA was extracted with the Qiagen RNeasy plant mini kit (Qiagen, Valencia, CA). Each RNA sample used for assessing gene expression was pooled from three 3-week-old plants. RNA quality and quantity were determined with an Agilent 2100 bioanalyzer (Agilent Technologies, Santa Clara, CA) before being labeled with an Ambion MessageAmp II-biotin enhanced labeling kit (Ambion ${ }^{\circledR}$; Invitrogen, Carlsbad, CA). Affymetrix GeneChip Tomato Genome Arrays (Affymetrix, Santa Clara, CA) were hybridized and scanned in the Array Core Laboratory at the Benaroya Research Institute, Seattle, WA, using a Genechip ${ }^{\circledR} 30007 \mathrm{G}$ scanner (Affymetrix) and Genechip ${ }^{\circledR}$ Fluidics station 450 (Affymetrix). Microarray data analysis was performed with the programs GCOS 1.4 (Affymetrix) and MapMan Version 3.5.1 (<http://mapman.gabipd.org $>$ ). Only genes that had signal strengths greater than

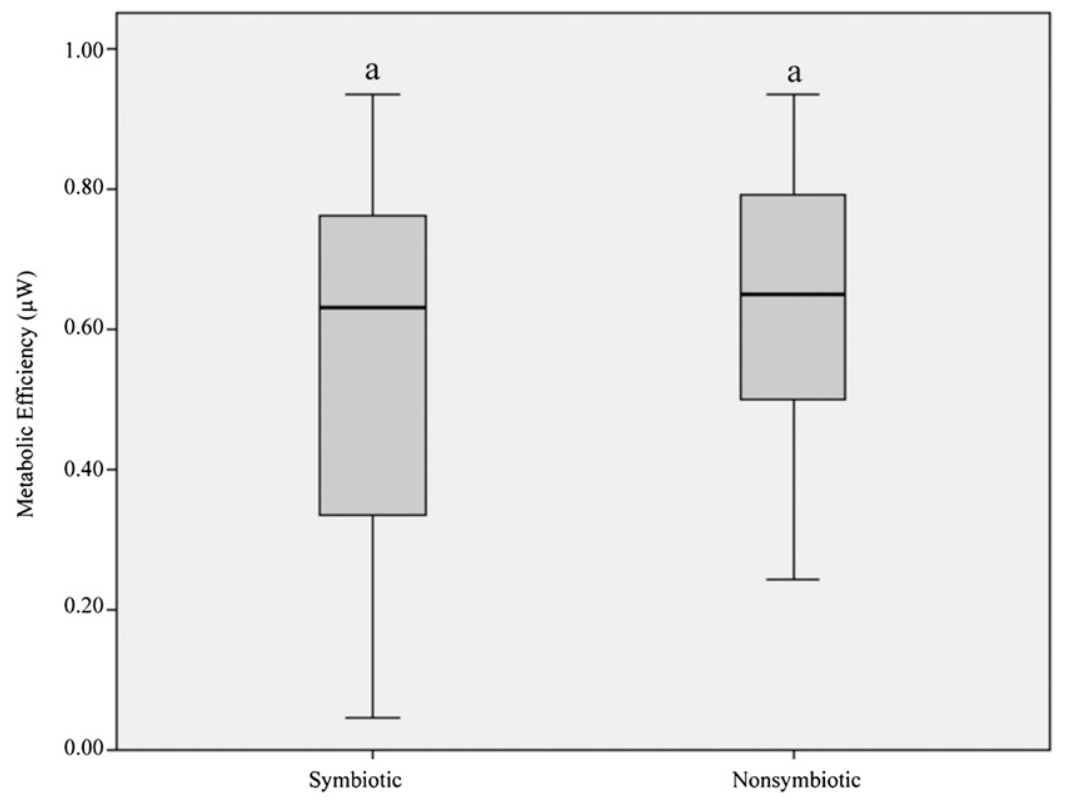

Fig. 2. Metabolic efficiency of symbiotic and nonsymbiotic rice seedlings $(\mathrm{N}=39)$ determined by differential scanning calorimetry. The horizontal bars indicate the medians. The vertical bars indicate the smallest and largest values. There is no significant differences between treatments $(t$ test, $P>0.05)$.

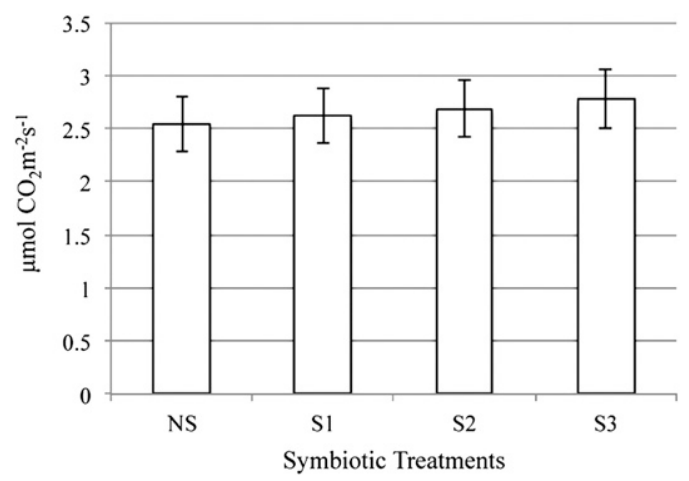

Fig. 3. Photosynthetic rates of symbiotic and nonsymbiotic tomato plants in the absence of stress. Each bar represents the average of nine replicates. No significant differences in photosynthetic rates were observed in symbiotic $(\mathrm{S} 1=$ FcRed1; $\mathrm{S} 2=$ F. redolens; $\mathrm{S} 3=$ Alternaria $\mathrm{sp}$.) vs. nonsymbiotic (NS) treatments $(P>0.1)$.
2.0 and had at least a threefold difference from the control were analyzed.

\section{RESULTS}

Symbiosis versus metabolic efficiency. Metabolic efficiency was tested on NS and stress with differential scanning calorimetry dividual rice plants revealed that although there was substantial variation between samples, there was no significant difference in (Fig. 2). Similar results were obtained with tomato (data not shown).

Because there was no difference in metaphotosynthetic activity and efficiency were amined. No differences were found in photosynthetic rate (Fig. 3) or maximum quantum $\mathrm{S}$ rice and tomato plants in the absence of sothermally. Analysis of 39 in- efficiencies of PSII reaction centers $(\mathrm{Fv} / \mathrm{Fm})$ (Maxwell and Johnson, 2000) between S and NS tomato plants without stress. However, there were significant differences in the quantum yield of photosynthetic electron transport through PSII (ФPSII) in plants that were symbiotic with FcRed1, Fc18, and CpVF (Fig. 4). This indicated that a greater proportion of the light absorbed by chlorophyll in $\mathrm{S}$ plants was being used in photosynthesis compared with NS plants.

Symbiosis versus plant gene expression. In NS plants exposed to salt stress or heat stress, an equivalent number of genes were up-expressed as were down-expressed (Table 1). To see if the genes being expressed in $\mathrm{S}$ plants were different from those in NS plants under the same conditions, the NS microarray was compared with the S plants treated with the same abiotic stress. In the absence of stress, plants that were symbiotic with FcRed1 had two times more genes upregulated than downregulated compared with unstressed NS plants. Once $250 \mathrm{~mm} \mathrm{NaCl}$ was applied to the plants, the number of upregulated genes in FcRed1 plants increased to more than five times the number that were downregulated. There was little change in the number of genes downregulated but a large increase in the number of genes with an increased expression level compared with the NS plants treated with $250 \mathrm{~mm} \mathrm{NaCl}$. In the absence of salt stress, gene expression patterns in Fc18-colonized plants was similar to FcRed1 plants with $\mathrm{S}$ plants having approximately two times the number of upregulated genes compared with NS plants. However, in the presence of salt stress, Fc18-colonized plants increased the number of upregulated to downregulated genes by more than sevenfold.

A very different gene expression pattern was observed in plants that were symbiotic with Cp4666D. In the absence of heat stress, plants had twice as many genes downregulated than the NS plants but when plants were exposed to heat stress, $\mathrm{S}$ plants had three times more upregulated genes than NS plants exposed to stress. CpVF-colonized plants had a similar gene expression pattern as NS plants in the absence of stress. However, in the presence of stress, CpVF plants had almost a sixfold increase in the number of upregulated genes compared with NS plants.

\section{DISCUSSION}

In the absence of stress, Class 2 fungal endophytes cause a significant increase in biomass and a reduction in water consumption of up to $50 \%$ in host plants (Redman et al., 2001, 2011; Rodriguez et al., 2008, 2009a). These results suggest that S plants are either more metabolically efficient or produce more photosynthate than NS plants. In the absence of abiotic stress, the effect of endophytes on plant metabolism appears to be the same regardless of the habitat of origin and stress tolerance conferred by the fungi. Habitat adapted (FcRed1 and Cp4666D) and non-adapted (Fc18 and $\mathrm{CpVF}$ ) endophytes 


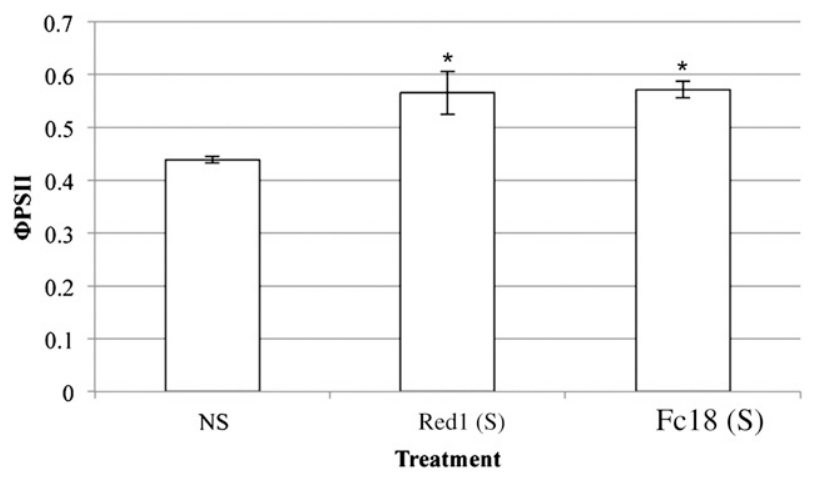

Fig. 4. Photochemical efficiency, $\Phi P S I I$, in symbiotic (S) and nonsymbiotic (NS) tomato plants. Measurements were collected with a LI-COR 6400 photosynthesis instrument. Each bar represents the average of five replicates. Plants were grown in the absence of salt stress. Asterisks indicate significant differences ( $t$ test, $P>0.05$ ) from NS control plants.

Table 1 . The effect of fungal symbiosis on gene expression in tomato with and without exposure to abiotic stress.

\begin{tabular}{|c|c|c|c|c|c|}
\hline \multirow[b]{2}{*}{ Expt. } & \multirow{2}{*}{$\begin{array}{l}\text { NS control } \\
\text { array }\end{array}$} & \multirow[b]{2}{*}{$\mathrm{S}$ array } & \multicolumn{2}{|c|}{ Number of genes changing expression } & \multirow[b]{2}{*}{$\mathrm{U}: \mathrm{D}$} \\
\hline & & & Upregulated (U) & Downregulated (D) & \\
\hline \multirow{5}{*}{$\begin{array}{l}\bar{E} \\
\text { Dू }\end{array}$} & NS- & FcRed1- & 94 & 47 & 2.0 \\
\hline & NS- & Fc18- & 162 & 69 & 2.3 \\
\hline & NS+ & FcRed1+ & 287 & 52 & 5.5 \\
\hline & NS+ & Fc18+ & 244 & 33 & 7.4 \\
\hline & NS- & NS+ & 142 & 144 & 1.0 \\
\hline \multirow{5}{*}{ 营 } & NS- & Cp4666D- & 114 & 267 & 0.4 \\
\hline & NS- & $\mathrm{CpVF}-$ & 157 & 121 & 1.3 \\
\hline & NS+ & $\mathrm{Cp} 4666 \mathrm{D}+$ & 175 & 58 & 3.0 \\
\hline & NS+ & $\mathrm{CpVF}+$ & 235 & 41 & 5.7 \\
\hline & NS- & NS+ & 246 & 207 & 1.2 \\
\hline
\end{tabular}

$(+)$ Stress treatment, $(-)$ control treatment. The NS array was used as the control in each case; therefore, the number of genes noted are genes from the $\mathrm{S}$ arrays (unless otherwise stated), which are upregulated or downregulated compared with the NS array.

$\mathrm{NS}=$ nonsymbiotic; $\mathrm{S}=$ symbiotic

increased plant growth rates, biomass, and the number of upregulated genes while decreasing water consumption. Moreover, there were no differences between $\mathrm{S}$ and NS plants with regard to metabolic efficiency or photosynthetic rates. These "non-stress"-related mutualistic plant benefits conferred by the endophytes may be common aspects of symbiotic communication between Class 2 endophytes and plant hosts.

It is noteworthy that in the presence of abiotic stress, non-adapted endophytes increase the number of upregulated plant genes significantly more than the habitatadapted endophytes. This suggests that both endophyte groups are communicating with plants in response to the stress and that qualitative differences in gene expression are responsible for stress tolerance.

Based on the combination of plant metabolic and gene expression responses to the endophytes, we propose the following working model to explain Class 2 endophyte conferred fitness benefits in plants (Fig. 1). The endophytes occupy the apoplastic spaces in plants but appear to penetrate into plant cells infrequently (Freeman and Rodriguez, 1993). Clearly, there is an active form of communication that occurs in planta and we surmise that the endophytes secrete effector molecules that are transported into plant cells. The effectors may be plant hormones made by the endophytes (Redman et al., 2011), signaling molecules, microRNAs, or other less defined biomolecules. The site of effector action may be cytoplasmic or in an organellealtering plant metabolism through one or both routes. Although some endophyte effectors may act on limited areas of a plant (e.g., growth promotion by meristem modification or root development), other effects are systemic such as increased quantum efficiency of PSII. Regardless, fungal endophytes have profound impacts on plant metabolism, fitness, and adaptation.

The sustainability of agriculture in the 21st century is being threatened by climate-induced changes in rainfall, minimum/maximum growing season temperatures, and increased occurrence of plant diseases (FAO, 2009a, 2009b, 2011; Sturrock et al., 2011). In addition, water recycling, salt water intrusion of aquifers, and increased frequency and severity of storms are increasing the salinization of soils. We propose a symbiogenic strategy for mitigating the impacts of abiotic stress in agriculture based on previous work demonstrating that endophytes are able to confer drought, salt, temperature, and disease tolerance to crop plants. One of the great benefits of symbiogenic technology is that it can be applied to most, if not all, crop species and symbiotic benefits are conferred within $36 \mathrm{~h}$ post-inoculation.
However, successful use of fungal endophytes in agriculture must overcome several challenges including 1) sufficient commercial production of the endophyte to meet demands; 2) extended shelf life for fungal viability and symbiotic function; 3) efficient inoculation of seed or seedlings; 4) compatibility with existing agricultural practices; 5) regulatory compliance; and 6) marketing and distribution. We have developed methods to overcome Challenges 1 to 4 and will be field-testing symbiogenic technology in the coming year. Although there is still more to learn about this technology, it is clear that it can address many current and emerging crop issues and contribute to sustainable agricultural production in this century.

\section{Literature Cited}

Apel, K. and H. Hirt. 2004. Reactive oxygen species: Metabolism, oxidative stress, and signal transduction. Annu. Rev. Plant Biol. 55:373-399.

Chaw, S., C. Chang, H. Chen, and W. Li. 2004. Dating the monocot-dicot divergence and the origin of core eudicots using whole chloroplast genomes. J. Mol. Evol. 58:424-441.

Criddle, R.S. and L.D. Hansen. 1999. Calorimetric methods for analysis of plant metabolism, 711763. In: Kemp, R.B. (ed.). Handbook of thermal analysis and calorimetry, Vol. 4: From macromolecules to man. Elsevier Science. Amsterdam, Netherlands.

Criddle, R.S., B.N. Smith, and L.D. Hansen. 1997. A respiration based description of plant growth rate responses to temperature. Planta 201:441-445.

FAO. 2009a. Coping with changing climate: considerations for adaptation and mitigation in agriculture. 22 Aug. 2011. <http://www.fao.org/ docrep/012/i1315e/11315e00.htm>. Food and Agriculture Organization of The United Nations.

FAO. 2009b. Profile for climate change. 22 Aug. 2011. <http://www.fao.org/docrep/012/11323e/ $\mathrm{i} 1323 \mathrm{e} 00 . \mathrm{htm}>$. Food and Agriculture Organization of The United Nations.

FAO. 2011. Adapt framework programme on climate change adaptation. 22 Aug. 2011. <http:// www.fao.org/docrep/014/i2316e/i2316e00.pdf>. Food and Agriculture Organization of The United Nations.

Flowers, T.J. and S.A. Flowers. 2005. Why does salinity pose such a difficult problem for plant breeders. Agr. Water Mgt. 78:15-24.

Freeman, S. and R.J. Rodriguez. 1993. Genetic conversion of a fungal plant pathogen to a nonpathogenic, endophytic mutualist. Science 260:75-78.

Hansen, L.D., R.S. Criddle, and B.N. Smith. 2005. Calorespirometry in plant biology, p. 17-30. In: Lambers, H. and M. Ribas-Carbó (eds.). Plant-Respiration: From cell to ecosystem. Springer, Dordrecht.

Márquez, L.M., R.S. Redman, R.J. Rodriguez, and M.J. Roossinck. 2007. A virus in a fungus in a plant-Three way symbiosis required for thermal tolerance. Science 315:513-515.

Maxwell, K. and G.N. Johnson. 2000. Chlorophyll fluorescence-A practical guide. J. Expt. Bot. 51:659-668.

Mayr, E. 1993. What was the evolutionary synthesis? Trends Ecol. Evol. 8:31-33.

Miglia, K.J., E.D. McArthur, R.S. Redman, R.J. Rodriguez, J.C. Zak, and D.C. Freeman. 2007. Genotype, soil type, and locale effects on reciprocal transplant vigor, endophyte growth, and microbial functional diversity of a narrow 
sagebrush hybrid zone in Salt Creek, Canyon, Utah. Amer. J. Bot. 94:425-436.

Perelman, S.B., E.J. Chaneton, W.B. Batista, S.E. Burkart, and R.J.C. Leon. 2007. Habitat stress, species pool size and biotic resistance influence exotic plant richness in the Flooding Pampa grasslands. J. Ecol. 95:662-673.

Redman, R.S., D.D. Dunigan, and R.J. Rodriguez. 2001. Fungal symbiosis: From mutualism to parasitism, who controls the outcome, host or invader? New Phytol. 151:705-716.

Redman, R.S., S. Freeman, D.R. Clifton, J. Morrel, G. Brown, and R.J. Rodriguez. 1999. Biochemical analysis of plant protection afforded by a nonpathogenic endophytic mutant of Colletotrichum magna. Plant Physiol. 119:795-804.

Redman, R.S., Y.O. Kim, C.J.D.A. Woodward, C. Greer, L. Espino, S. Doty, and R.J. Rodriguez. 2011. Increased fitness and adaptation of rice plants to cold, drought and salt stress via habitat adapted symbiosis: A strategy for mitigating impacts of climate change. PLoS One 6:E14823.

Redman, R.S., K.B. Sheehan, R.G. Stout, R.J. Rodriguez, and J.M. Henson. 2002. Thermotolerance generated by plant/fungal symbiosis. Science 298:1581.

Rodriguez, R.J., D.C. Freeman, E.D. McArthur, Y.O. Kim, and R.S. Redman. 2009a. Symbiotic regulation of plant growth, development and reproduction. Commun. Integr. Biol. 2:1-3.

Rodriguez, R.J., J.F.J. White, A.E. Arnold, and R.S. Redman. 2009b. Fungal endophytes: Diversity and functional roles. New Phytol. 182:314-330.

Rodriguez, R.J., J. Henson, E. Van Volkenburgh, M. Hoy, L. Wright, F. Beckwith, Y. Kim, and R.S. Redman. 2008. Stress tolerance in plants via habitat-adapted symbiosis. International Society of Microbial Ecology. 2:404-416.

Stout, R.G. and T.S. Al-Niemi. 2002. Heat-tolerance flowering plants of active geothermal areas in
Yellowstone National Park. Ann. Bot. (Lond.) 90:259-267.

Sturrock, R.N., S.J. Frankel, A.V. Brown, P.E. Hennon, J.T. Kliejunas, K.J. Lewis, J.J. Worrall, and A.J. Woods. 2011. Climate change and forest diseases. Plant Pathol. 60:133-149.

Tester, M. and A. Bacic. 2005. Abiotic stress tolerance in grasses. From model plants to crop plants. Plant Physiol. 137:791-793.

Wolfe, K.H., M. Gouy, Y. Yang, P.M. Sharp, and W. Li. 1989. Date of the monocot-dicot divergence estimated from chloroplast DNA sequence data. Proc. Natl. Acad. Sci. USA 86:6201-6205.

Yamaguchi, T. and E. Blumwald. 2005. Developing salt-tolerant crop plants: Challenges and opportunities. Trends Plant Sci. 10:615-620.

Yang, Y.W., K.N. Lai, P.Y. Tai, and W.H. Li. 1999. Rates of nucleotide substitution in angiosperm mitochondrial DNA sequences and dates of divergence between Brassica and other angiosperm lineages. J. Mol. Evol. 48:597-604. 